\title{
HEAD INJURY AS A CAUSE OF INTERNAL CAROTID THROMBOSIS *
}

\author{
BY \\ C. B. SEDZIMIR \\ From the Regional Neurosurgical Centre, Walton Hospital, Liverpool
}

Thrombosis of the internal carotid artery with neurological signs may develop after direct violence to the wall of the artery in the neck. This has been known at least as far back as the last century and calls for little comment. A description of two such cases was published in 1936 by Caldwell, who warns against the error of attributing to concomitant head injury the symptoms and signs which develop as a result of traumatic thrombosis of the internal carotid artery in the neck.

Ramsay Hunt first called attention to the occurrence of spontaneous thrombosis of the internal carotid artery in 1914. He and most successive authors conclude that arteriosclerosis is the most important aetiological factor. In this communication evidence is produced and the mechanism suggested whereby, in some cases of internal carotid thrombosis, a major or minor head injury may be instrumental in precipitating the thrombotic process. The clinical evidence of such a thrombosis may be immediate or delayed and in the latter event may present, some time after the injury, as a case of "spontaneous" thrombosis.

Of the other known aetiological factors, thromboangiitis obliterans has been discussed by Davis and Perret (1947) and Cloake (1951). Thrombosis occurring after rupture of an intracranial aneurysm has been recorded. Only two cases are established in the literature in which a thrombotic process resulted from a head injury. One of these cases was described by the late Sir Hugh Cairns (1942) and he quoted another case under the care of Löhr. It is of interest that in these cases thrombosis occurred in the intracranial segment of the artery. The present series consists of six such cases; in five, a major head injury, and in one, minor trauma to the skull, are held responsible for the development of thrombosis of the intracranial segments of the artery.

\section{Case Reports}

Case $1(4717 / 53)$. - A woman, aged 54, was admitted unconscious following a road accident. She had a

* A shortened version of this paper was read before the Society of British Neurological Surgeons in December, 1954. haematoma of the left orbit with chemosis. There were no abnormal neurological signs. On the following day a cerebrospinal fluid rhinorrhoea was noticed which subsided in two days. On the fifth day after the injury she was conscious and capable of sensible conversation and again no abnormal neurological signs were elicited. On the seventh day she became drowsy. Within two hours she developed dysphasia and paresis of the right arm. Shortly afterwards she became unconscious. She was transferred to the Neurosurgical Unit and the usual three sets of burr-holes were made. On the left side about $20 \mathrm{ml}$. of yellow subdural fluid was aspirated. The brain was a few millimetres away from the dura on both sides and the intraventricular pressure was subatmospheric. The left hemisphere felt somewhat softer than the right to the exploring cannula. On lumbar puncture cloudy fluid containing pneumococci was found. The meningitis was vigorously treated but she continued to deteriorate : the right leg became paralysed as well as the right arm, and she died 13 days after the injury.

Necropsy.-A little purulent exudate was found in the cisterna ambiens. There was a linear fracture through the left cribriform plate extending posteriorly across the base of the left anterior clinoid process and also down into the body of the sphenoid. The dura over the cribriform plate showed a minute tear. The frontal and ethmoidal sinuses were healthy, but there was a small amount of mucopus in the sphenoidal sinus.

The left hemisphere was softened. The basilar, middle cerebral, and both internal carotid arteries showed a moderate degree of atheroma. The left internal carotid artery was thrombosed throughout the length of its intracranial portion as far as its bifurcation. At the point where the artery pierced the dura of the cavernous sinus the thrombus was firmly adherent to the wall at the site of an atheromatous plaque. Above and below this point the thrombus was softer and only loosely filled the lumen. There was no thrombus in the carotid arteries in the neck. Microscopic section at the site of adherence of the thrombus did not show any sign of infection in the arterial wall.

Case 2 (5700/54).-A man, aged 42, fell off a moving bus. He was unconscious, restless, but had no obvious neurological signs. There were minor facial injuries and orbital haematomata. A cerebrospinal fluid rhinorrhoea was noticed. Two days later he became more 
deeply comatose and a right hemiparesis was discovered. He was transferred to the Neurosurgical Unit and diagnosed as having an internal carotid thrombosis but exploratory burr-holes were made and excluded intracranial haemorrhage. He died 24 hours later.

Necropsy.-The skull showed a fracture similar to that described in Case 1. There was no infection. The left hemisphere was soft. The left internal carotid artery was occluded by a thrombus throughout the intracranial segment and this extended into the neck to a point $5 \mathrm{~cm}$. above the carotid sinus. Distally the thrombus entered the left middle and anterior cerebral arteries. Both internal carotid arteries showed patches of atheroma in the region of the carotid sinus but there was no sign of thrombus formation there. The peripheral vessels, heart, and all other organs were normal. There was, in particular, no evidence of primary thrombus formation from which an embolus could have originated.

Case $3(5850 / 54)$.- Eight years before, at the age of 32, this man fell and hit the left side of his head. He was not unconscious and after a time he continued his work. He went to bed that night feeling well but when he woke up the next morning he found the left side of his body paralyzed. Later he noticed anosmia and became aware of inability to see objects on the left side. In the course of a few months the leg partially recovered and he could walk. There was no recovery in the arm or in the hemianopia. On admission to the Neurosurgical Unit, eight years after the accident, right carotid angiography showed the characteristic arrest of the dye in the internal carotid artery $1.5 \mathrm{~cm}$. above its origin. Left carotid angiograms showed filling of the left cerebral tree and also filling of the right anterior and middle cerebral arteries, the latter being only scantily visualized.

Case 4.-This patient was under the care of Mr. R. A. Money, of Sydney, Australia, who kindly provided the clinical data and permitted the publication of the case in this series.

A man, aged 32, was involved in a car collision in November, 1952. He received frontal injuries with a laceration of the forehead and lost consciousness. When he recovered he walked half a mile for help. He was then admitted to a local hospital and was found to be quite rational. The next day his condition deteriorated, he became unconscious, and developed, in the course of a few hours, a left hemiplegia. Five days after the injury he was admitted under the care of Mr. R. A. Money. He was stuporose and had a complete flaccid left hemiplegia with impairment of sensation on that side. An electroencephalogram indicated marked damage to the right side of the brain and suggested that a subdural haematoma might be present. Angiography by percutaneous and cut-down methods showed a complete obstruction of the right internal carotid artery about 1 in. above the carotid sinus. He was given heparin and a series of stellate ganglion blocks were performed without giving neurological improvement. During 1953 sufficient power had returned to the left leg to allow him to walk with an appliance. In April, 1953, he had an epileptic attack and fits recurred at odd intervals. An electroencephalogram in 1954 revealed slow waves originating from the right hemisphere. There was no further recovery in the limbs.

Case 5 (5053/53). - A woman, aged 69, was knocked down by a bicycle in August, 1953. She lost consciousness for about 15 minutes. She was admitted to the local hospital within 30 minutes of the accident where she was found to be slightly confused, the only abnormal neurological sign being a.left facial weakness. The next day her conscious level deteriorated and she developed a left hemiparesis. An epileptic fit was reported. She was admitted to the Neurosurgical Unit on the third day after her injury.

On admission she was conscious but disorientated and emotionally labile. The pulsation of the right temporal artery was more pronounced than on the left. She had bilateral orbital haematomata and a laceration over the left eyebrow. The left pupil was slightly larger than the right but reacted to light. The optic fundi were normal but she showed an attention defect in the left homonymous fields. She had a left facial weakness and a complete left hemiplegia, the tone being increased to a greater extent in the arm than in the leg. She was unable to find the left limbs without looking for them and she had a complete astereognosis, tactile inattention, and transposition of painful stimuli into the space in front of her. A diagnosis of right internal carotid thrombosis was made. She made slow but gradual progress and on discharge, some weeks later, the power of the left limbs was nearly normal although the other abnormal signs persisted. For humanitarian reasons, the very great temptation to perform angiography was resisted. The diagnosis remains clinical.

Case 6 (4662/53). - A woman, aged 28, received a severe left frontal and temporal head injury with coma, bilateral proptosis, and orbital haematomata. A loud bruit was audible over the left eye and could be abolished by compression of the left carotid artery. A left-sided extradural haematoma was evacuated without any improvement in her condition. A few hours later the bruit was no longer present and she developed a right hemiplegia. She died shortly afterwards and post-mortem examination was refused. The clinical evidence is very strong indeed, however, that thrombosis of the internal carotid artery followed upon a traumatic arteriovenous fistula within the cavernous sinus.

\section{Clinical Considerations}

These cases provide interesting material for speculation upon the clinical progress of some confusing head injuries. A patient, after a lucid interval, may show deterioration in the conscious level accompanied by the onset of focal signs. Exploration for massive intracranial haemorrhage may prove negative. The brain is not under tension ; on the contrary, it may appear atrophic or one hemisphere may be softer than the other. The patient may improve and be left with some greater or lesser disability. The diagnosis made may be one 


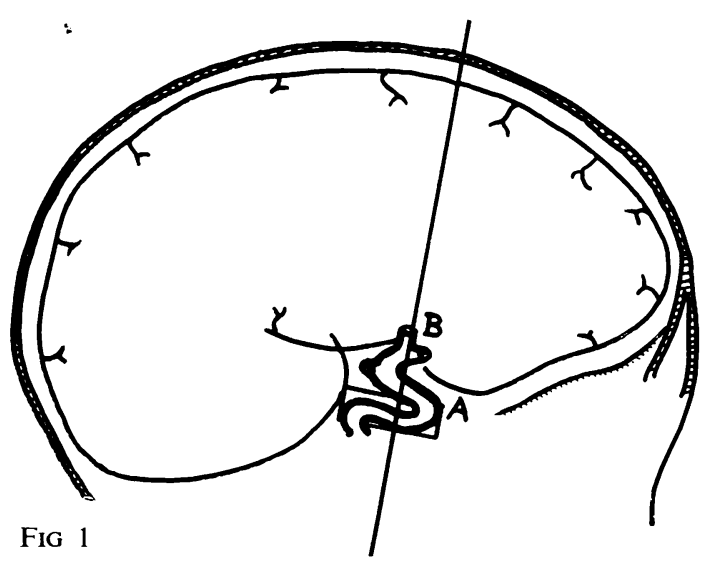

of post-traumatic apoplexy or thrombosis of the middle cerebral artery or one of its branches, particularly if the patient is elderly. Internal carotid thrombosis, which is possibly the real cause, will not be diagnosed.

\section{Anatomical and Pathological Considerations}

Analysis of the foregoing cases suggests that thrombosis of the internal carotid artery took place as a result of trauma to the intracranial segment and in the two cases examined after death a fracture of the floor of the anterior fossa was found in close proximity (similar to the case described by Cairns). In all cases the main impact was frontal. Particular attention is drawn to the fact that in Case 1 the thrombus was firmly adherent to the wall at the point of emergence of the artery from the cavernous sinus. Here the artery is anchored by the embracing dura mater. This point, too, marks the centre of a variable "syphon", the distal end of which curves backwards and upwards and forwards before the artery ends by dividing into the anterior and middle cerebral arteries. These soon become fixed to the brain (Fig. 1). It were as though nature provided this slack segment to take the stress should the artery at any time be subject to movement.

From experimental work, and other observations, we know that when a force is applied to the head there is a certain amount of movement of the brain within its coverings. It may be a violent movement, as in severe injuries, or of lesser degree in minor injuries. It would appear, therefore, that the distal segment of the internal carotid artery moves with the brain, to which it is tethered by its terminal branches, while the more proximal end remains immobile at the point where the artery leaves the cavernous sinus (Fig. 2). It is submitted that at this latter point the artery is subject to shearing strains. Such strains, more so if the artery is

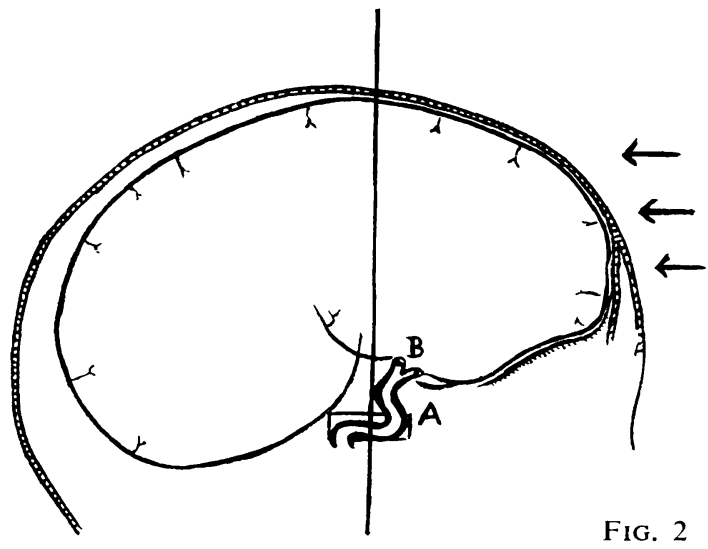

sclerotic, may well be the precipitating factor in the formation of a thrombus. In the elderly patient with some cerebral atrophy, it is reasonable to suppose that the range of cerebral movement would be increased. Any strain to which the artery may then be subjected would likewise increase, and would occur at a time when the ageing vessels are less able to compensate for such a strain.

It seems highly probable that, particularly in the younger patient, the same mechanism may induce a spasm of the internal carotid artery which, for a time, may mimic a true thrombosis both clinically and on angiography. Connolly (1954) told of such a case under his care. The same interpretation was applied to one patient in this unit who unfortunately was referred too late for the angiography to be conclusive.

\section{Discussion}

Cloake (1951) states "... There are two sites of thrombosis, one near the point of origin of the vessel, the other in the intracranial portion close to its termination." Fisher (1951) believes that the first portion of the internal carotid artery is particularly liable to atheroma and that spontaneous thrombosis occurs just distal to the carotid sinus. The commonest site of internal carotid thrombosis in cases reported in the literature appears to be in the neck, particularly if the diagnosis is made by angiography. It is also probable that a thrombosis which begins in the intracranial segment might sometimes spread proximally to the region of the bifurcation. This has been reported in the literature following rupture of an aneurysm of the internal carotid artery, and two cases have been encountered in this Unit. Such a retrograde spread is finally arrested at about 1 to $2 \mathrm{~cm}$. above the carotid sinus, because of the pounding of the blood as it whirls in and out from the sinus. This produces the 
familiar angiographic appearances in those who survive. The thrombosis may not reach this level, however, before death occurs.

When there is thrombosis of the internal carotid artery after a head injury there may be early clinical evidence of it. Alternatively, for reasons of circulatory dynamics and anatomical channels of collateral circulation, such evidence may not be apparent for weeks, months, or years afterwards, the trauma being forgotten or discounted. In addition to the six cases described here, 12 other cases of internal carotid occlusion were collected in this hospital. Two of these followed rupture of a berry aneurysm, two were embolic, and eight were grouped as "spontaneous". Two of the latter gave a clear history of head injury 10 months and 10 years before the onset of signs.

\section{Summary}

Six cases are reported of carotid thrombosis and hemiplegia occurring as a delayed complication of head injury.

The possible mechanisms are discussed.

The primary site of thrombosis may more commonly occur in the intracranial segment than was previously supposed and may spread in a retrograde fashion into the neck.

Delayed symptoms after a head injury with angiographic evidence of an apparently primary cervical occlusion may give rise to a diagnosis of "spontaneous" internal carotid thrombosis.

Some confusing clinical syndromes following a head injury and suggesting massive intracranial haemorrhage but with negative surgical findings may be explained by thrombosis of the internal carotid artery.

I wish to express my grateful thanks to Mr. A. S. Kerr, senior surgeon of this Unit, for his encouragement and helpful suggestions. I am indebted to him and to Mr. R. H. Hannah for permission to publish cases under their care. I am also indebted to Dr. M. Wodzinski for the post-mortem examinations.

\section{REFERENCES}

Cairns, H. (1942). Lisboa méd., 19, 375.

Caldwell, J. A. (1936). Amer. J. Surg., 32, 522.

Cloake, P. C. P. (1951). In Modern Trends in Neurology, p. 466, ed. A Feiling. Butterworth, London.

Connolly, C. (1954). Soc. Brit. Neurol. Surg., Belfast. Discussion.

Davis, L., and Perret, G. (1947). Brit. J. Surg., 34, 307.

Fisher, M. (1951). Arch. Neurol. Psychiat., Chicago, 65, 346.

Hunt, J. Ramsay. (1914). Amer. J. med. Sci., 147, 704.

\section{Addendum}

Since this paper was submitted for publication, a further case has been under treatment, a woman, aged 51, who sustained a frontal head injury with a fractured base, which gave rise to a thrombosis of the intracranial segment of the left internal carotid artery four days after the injury. The position of the thrombus and of the fracture were verified at necropsy. 